\title{
Reproductive functions of sows under the influence of vitamin-mineral supplement in the diet
}

\author{
A.A. Ovchinnikov", L.Yu. Ovchinnikova, and Yu.V. Matrosova \\ South Ural SAU, Troitsk, Chelyabinsk region, Russia
}

\begin{abstract}
. usage of feed additive with $35 \mathrm{mg} / \mathrm{head}$ per day folic acid and trace elements complex in a dose of $10-50 \mathrm{mg} / 100 \mathrm{~kg}$ of live weight, Hexavit multivitamin $196 \mathrm{mg} / \mathrm{head}$ per day and similar trace elements, as well as all studied supplements in the diets of three groups of enceinte sows showed that dietary supplements activate anabolic processes in the body aimed at fetuses development and the deposition of reserve nutrients in the mother's body. As a result, sows receiving the studied supplements gave more piglets by $18.6 \%$, more good piglets to weaning - by $4.3 \%$, in the cost structure the total costs decreased by $9.7-18.5 \%$. The complex use of all biologically active additives was not effective during the entire physiological period of pregnancy. With this, the following application scheme proved to be the most acceptable for metabolic processes' stimulation in body: joint in the first 84 days of pregnancy and in the last 30 days - only folic acid with trace elements.
\end{abstract}

\section{Introduction}

A century and a half have passed since the discovery of the first vitamins by Russian scientist Lunin, today vitamins have become an integral part of full-fledged feed composition for all types of agricultural animals and poultry also having other dietary supplements in their composition [1-3]. If there is a lack of vitamins in the diet or its absence, various kinds of pathologies arise in all physiological systems, metabolism, which imposes a negative imprint on the growth and development of animals, reproduction functions, livestock safety, and production profitability.

The group of biologically active substances include many chemical elements of the second period of the periodic system by D.I. Mendeleev. The participation of trace elements in redox and hydrolytic processes in the body, the need for the synthesis of soft and hard tissues, secretory function of reproduction organs, allows to consider them as integral components of full body nutrition [4-7].

\footnotetext{
*Corresponding author: tvi_t@@mail.ru
} 
As a rule, both vitamins and minerals are part of the premix in the production of fullfledged feed, the rate of which is up to $1.0 \%$ of feed weight. However, various mineral salts of trace elements can have a negative effect on the vitamins' activity, lower it or even neutralize the biological effect. In this case, the correction of the diet for a scarce food element is required by adding an additional amount of it. Similarly, mineral elements can reduce their expected biological effect.

Therefore, the aim of our research was to compare the reproducible functions of sows receiving various additional vitamin-mineral supplements in the diet. The tasks of research were to analyze the animals' feeding diet, the difference in the number of newborn piglets and their number to weaning, to compare quantitative indicators of metabolic processes in the body and changes in the economic performance of piglets' production during the preweaning period.

\section{Material and methods of research}

The aim and objectives of the research were realized at the pig breeding complex with industrial technology with modern conditions of feeding and keeping of the breeding stock. Four groups of large white breed enceinte sows inseminated with bores of planned breeds (landrace $\mathrm{x}$ duroc) kept by 15 heads.

Against the background of the standard full-fledged feeding diet of the second experimental group sows, they were additionally fed with a supplement of folic acid (35 $\mathrm{mg} / \mathrm{head}$ per day and trace elements salts of cobalt, copper, zinc, manganese at a dose of $10-50 \mathrm{mg}$ per $100 \mathrm{~kg}$ of body weight), III experimental - the main diet and vitamin supplement Hexavit in the amount of $196 \mathrm{mg} / \mathrm{head}$ per day, as well as trace elements salts as in animals of the II group, IV experimental group - folic acid, Hexavit and trace elements salts in the studied dosage.

Reproductive functions were evaluated by multifertility, large piglet fetuses, preservation of livestock by weaning age.

The live weight of piglets was controlled by individual weighing of each piglet from sows of control and experimental groups.

Morpho-biochemical indices of sows' blood were compared according to the study results in the first $2 / 3$ and in the last third of the pregnancy in five heads of each group.

Economic indicators were determined through calculation by the reported data of feed consumption and the number of piglets at the age of 28 days.

The results of the research were processed biometrically with the reliability level determination.

\section{Research results and discussion}

Nutritional value of fodder received by the breeding stock and their piglets corresponded to the qualitative parameters of the formulation, both in general nutritional value and for individual elements that characterize organic and vitamin-mineral part of full-fledged feed. During pregnancy and sucking period, feed consumption depended on interior indicators and the number of piglets, which in turn affected economic valuation indicators.

Characteristics of metabolic processes in the body of pregnant sows showed the most increased metabolism in the last two experimental groups of animals (Figure 1). 


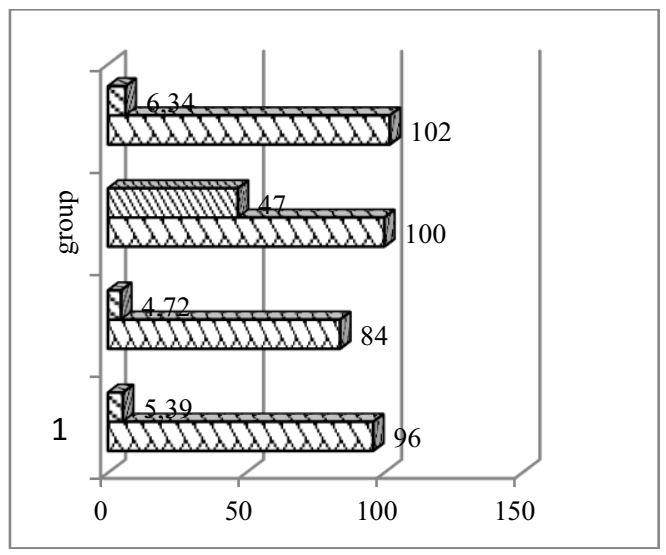

a)

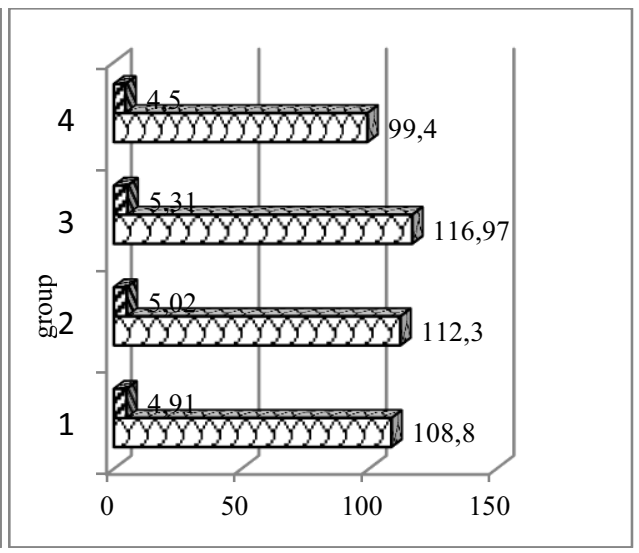

b)

Fig. 1. The content of hemoglobin $(\mathrm{g} / \mathrm{l})$ and erythrocytes $\left(10^{12} / \mathrm{l}\right)$ in the blood of sows in the 84 days period (fig. a) and in the last 30 days (fig. b) of pregnancy

Higher transport function of erythrocytes associated with metabolism was observed in animals of the latter group. Compared to the control group, this difference amounted to $17.6 \%$ in the first three-quarters of pregnancy, and in the last third it was at the level of the control group, while hemoglobin was higher than the control by $6.3 \%$ and inferior to the last before farrowing by $8.6 \%$. However, with the increase in the pregnancy period in sows of the first two experimental groups, there was a tendency to increase hemoglobin (3.2 and $7.3 \%, \mathrm{P}>0.05)$ and erytrocytes in the blood $(2.2$ and $8.1 \%, \mathrm{P}>0.05)$. It is characteristic to note the decrease in the defenses of sows' bodies (its cellular immunity) in animals with the study supplement in comparison to the control group. This difference amounted to $24.5-$ $31.0 \%(\mathrm{P} \leq 0.05)$ in the last experimental group, $19.4-25.8 \%$ - in the third, $17.7-15.2 \%$ $(\mathrm{P} \leq 0.05)$ - in the second experimental group.

Likely, the body immune defense of experimental groups' sows took place at the expense of humoral immunity and this issue deserves further separate study.

In many ways, comparison of individual markers of the main metabolism types allows to explain the material changes occurring in the body under the influence of the studied factor. For protein metabolism, the most widespread marker is total protein and urea, which is part of the residual nitrogen group. Their ratio in animals for periods of physiological state is shown in Figure 2.

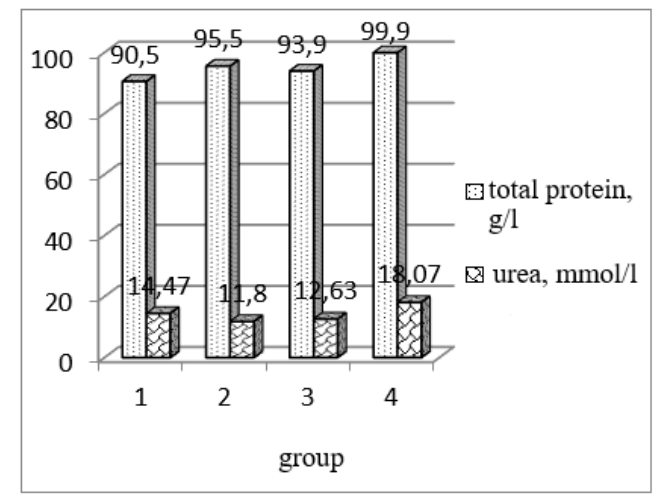

a)

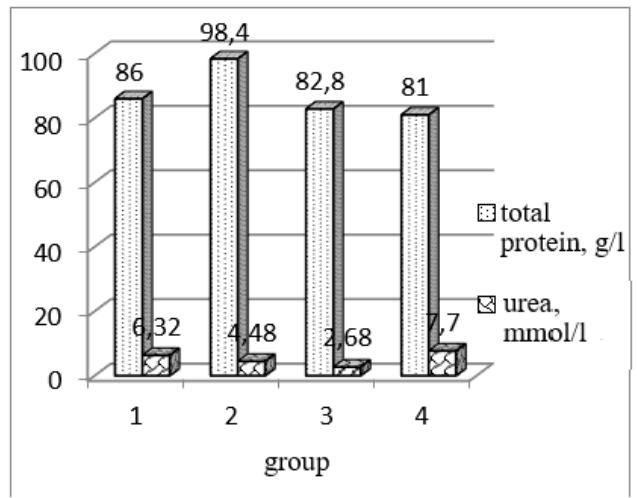

b)

Fig. 2. The content of total protein and urea in the blood serum of sows in the first (a) and the last third of pregnancy (b). 
With the increase in animals' pregnancy, an increase in total protein in the blood of experimental groups' sows under the influence of the bioactive component was characteristic. At the same time, there was a better use of the nitrogenous feed part confirmed by a decrease in blood urea, reliable in the second and third group (18.5 and $12.7 \%, \mathrm{P} \leq 0.05$ ), only the trend of this change was observed in the third group.

With the approximation of sows' farrowing, the protein exchange of experimental groups has changed; the total protein content in the last two groups decreased by 3.7 and $5.8 \%(\mathrm{P} \leq 0.001)$ in comparison with the control group, while its content increased in the second control group by $14.4 \%(\mathrm{P} \leq 0.001)$. However, only in the second and third group nitrogenous substances were reliably better used $(\mathrm{P} \leq 0.001)$, which affected the reproduction of animals.

The second marker characterizing metabolism are common lipids and cholesterol (Figure 3).

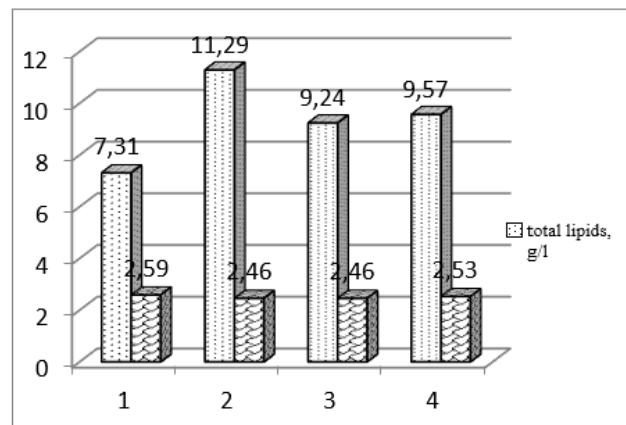

a)

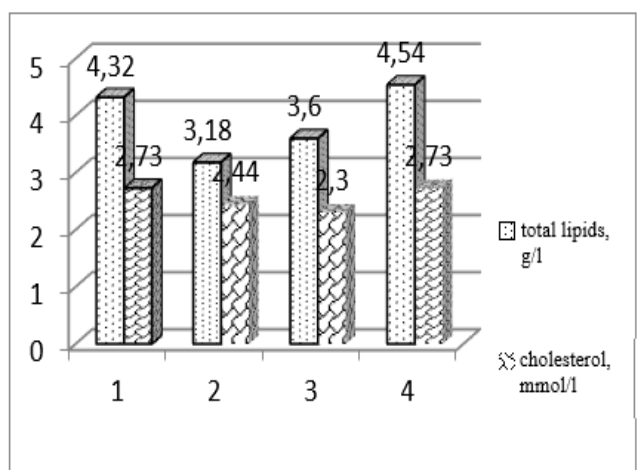

b)

Fig. 3. The content of total lipids and cholesterol in sows' blood by pregnancy periods (a - the first two-thirds, $b$ - the last third).

Vitamin-mineral supplement in the diet of sows stimulated lipid metabolism during this period of pregnancy. As a result, the level of total lipids in the sows' blood of the II group was higher than the I control by $51.4 \%$ (P $\leq 0.05)$, group III and IV - by $26.4-30.9 \%$.

Considering that during this pregnancy period there is fetus formation and main tissues and organs formation, a positive feature is the increase in energy metabolism in the blood of experimental groups' animals, confirming an increase in the level of glucose in whole blood (Figure 4).

This fact indicates a more intense energy metabolism in the body of enceinte animals, but most intensely proceeded under the influence of vitamins and trace elements.

These biologically active substances in the diet of the experimental groups' sows did not affect mineral metabolism, in particular, the level of calcium and phosphorus in the blood. Their ratio was within the physiological norm. 


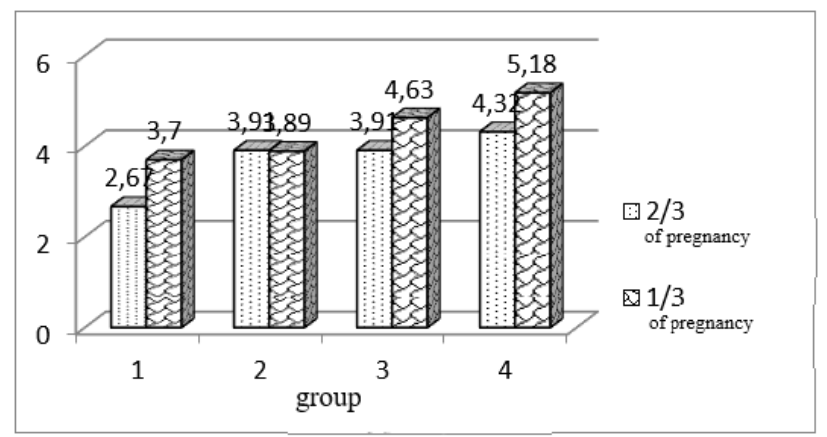

Fig. 4. Blood glucose content of sows.

Metabolism has a direct effect on the trophism of the fetuses, growth, and development of fetal membranes. Proper use of dietary supplements in the diet of enceinte sows can reduce fetus mummification, stillborn piglets' birth, thereby directly affect reproduction functions of sows.

From a production point of view, an important point is to consider the total number of piglets born, which indicates the actual number of fertilized eggs without regard to those resolved under the influence of various reasons. The number of living and stillborn is calculated from the number of born piglets, the cause of the latter should be specified individually.

The graph (Figure 5) reflects the sows' multifertility of control and experimental groups, which allows to make a certain conclusion.

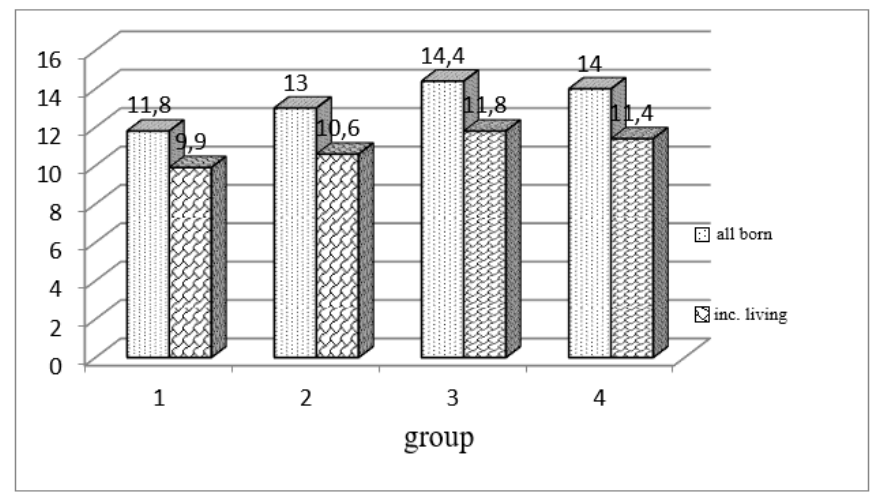

Fig. 5. Number of all born and living piglets in the nest, heads.

A comparison of the reproducible functions of sows receiving vitamin-mineral feed additive showed the difference in the total number of piglets born. Animals of experimental groups outperformed the control in total number of litter by $10.2 \%, 22.0$ and $18.6 \%$, live by 0.7 heads, 1.9 and 1.5 heads. Vitamin-mineral additive did not affect the litter's large fetuses; on average, piglets had a weight of $1.26-1.36 \mathrm{~kg}$.

However, changes in the physiological status of the organism of the newborn young, which occurred because of the inclusion of vitamins and minerals into the mother's diet, affected the body's defenses hence the safety of young (Figure 6). 


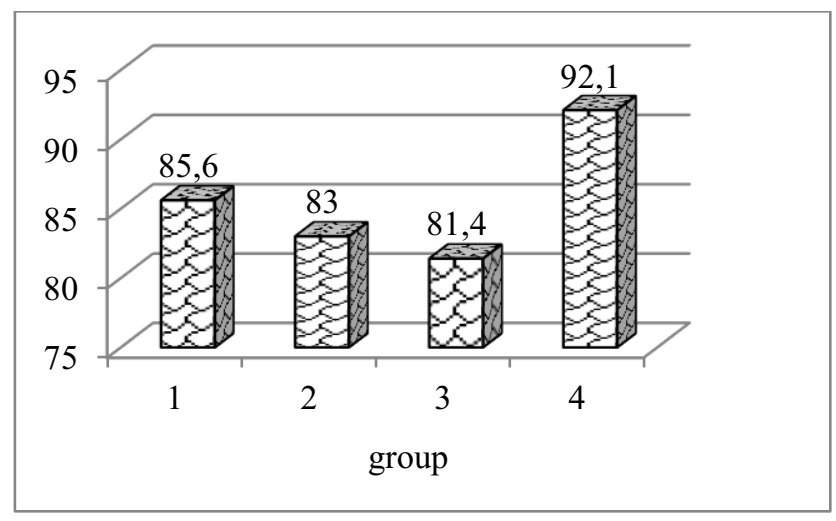

Fig. 6. Preservation of piglets during the preweaning period, $\%$.

Of all the experimental groups, only the last showed higher livestock preservation than the control by $6.5 \%$, in the other experimental groups - lower by 2.6 and $4.2 \%$.

This difference affected the economic efficiency assessment of feed additives use from this set of vitamins and trace elements (Fig. 7).

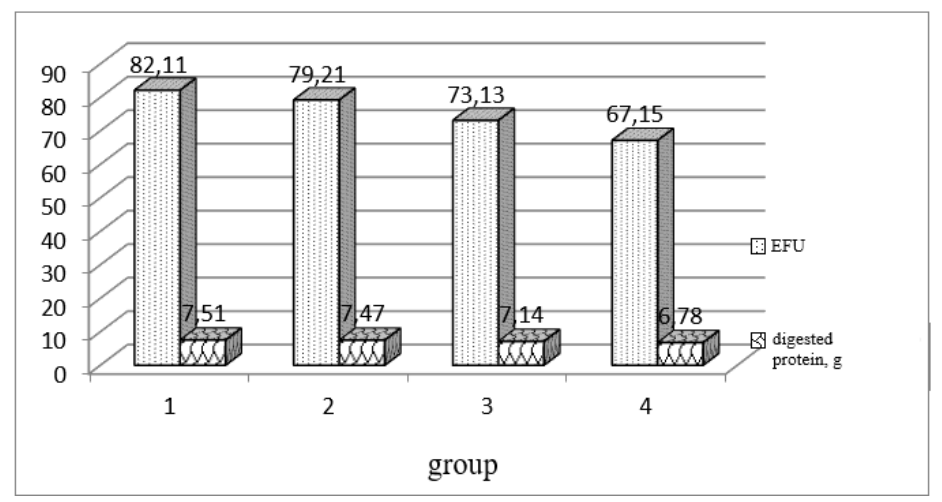

Fig. 7. Costs of EFU and digested protein per one good piglet.

In comparison with the analogues of the control group, the combined use of all tested additives in the sows' diet allowed to reduce feed costs by $9.7-18.5 \%$, in other groups this difference was lower - $0.5-11.1 \%$.

Earlier studies by S.V. Burtseva and I.A. Pushkarev [8] when feeding enceinte sows with vitamin supplement "LipoKar" at a dose of 1,1-2,1 g per head per day in the last three weeks of pregnancy coincide with the above data. As a result, the sows' multifertility of those researchers increased by $1.7-10.3 \%$, preservation - by $1.6-6.8 \%$, as well as the size of piglets at birth - by $20.0 \%$.

A positive correlation dependency was established between the use of vitamin supplement Vetvital B in the diet of pregnant sows and their economic and biological indicators that allows to receive livestock of young pigs with predicted traits of meat quality [9]. A similar result in the change in nutrients' digestibility in pigs in breeding and fattening was obtained when combining vitamin-mineral additive Kostavit forte in the animal feed. This biologically active complex positively affected the productivity of pigs and profitability of pork production [10].

However, there is evidence recommending the use of folic acid in the diets of sows in the first nine weeks of pregnancy [11]. At the same time, there was also an increase in the reproducible functions of sows. This vitamin not only has a positive effect on reproduction 
functions, but also on the hematopoietic function of the body, as well as protein metabolism.

\section{Conclusion}

We recommend using the folic acid supplement with Hexavit and biogenic trace elements in the diet of sows in the first two-thirds of pregnancy, in the last third - only folic acid with trace elements, which is enough to stimulate and increase the reproduction functions in the body, the preservation of sucking period piglets, cultivation and reduction of feed costs to bring them to weaning age.

\section{References}

1. V.M. Golushko, Actual problems of intensive pig breeding development: mat. coll. of scientific-practical conference of Bryansk SAU, 136-144 (2020)

2. A.A. Ovchinnikov, L.Yu. Ovchinnikova, Yu.V. Matrosov, E.M. Yermolova, D.A. Konovalov, Revista inclusions, 7(S2-2), 168-176 (2020)

3. R.R. Fatkullin, A.A. Ovchinnikov, E.M. Ermolova, Y.V. Matrosova, Int. Journal of Engineering \& Technology 7(3.14), 300-304 (2018)

4. A.V. Kravchenko, V.M. Golushko, S.A. Linkevich, Actual problems of intensive pig breeding development: mat. coll. of scientific-practical conference of Bryansk SAU, 159-164 (2020)

5. L.A. Morozova, Scientific and innovative technologies as a factor of sustainable development of agro-industrial complex: collection of articles on mat. of All-Russ. (national) scientific-practical. conf. of Kurgan SAA, 116-120 (2020)

6. N.A. Pozdnyakova, Scientific and innovative technologies as a factor of sustainable development of agro-industrial complex: collection of articles on mat. of All-Russ. (national) scientific-practical. conf. of Kurgan SAA, 152-156 (2020)

7. M.G. Chabaev, Actual problems of intensive pig breeding development: mat. coll. of scientific-practical conference of Bryansk SAU, 205-208 (2020)

8. S.V. Burtseva, I.A. Pushkarev, Bulletin of Altai SAU 4 (162), 118-120 (2018)

9. S.F. Sukhanova, N.A. Pozdnyakova, A.L. Zasypkin, Bulletin of Kurgan SAA. 2, 47-51 (2019)

10. O.Yu. Yunusova, L.V. Sycheva, R.V. Malchikov, Bulletin of Meat Cattle 1 (97), 107111 (2017)

11. A. V. Solyanik [et al.], Use of biologically active substances to increase the productivity and natural resistance of sows: monograph, 179 (2002) 